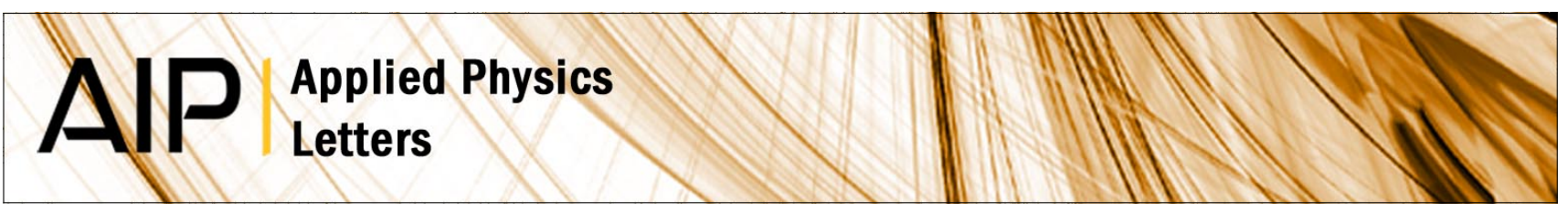

\title{
Counting molecular-beam grown graphene layers
}

Annette S. Plaut, Ulrich Wurstbauer, Aron Pinczuk, Jorge M. Garcia, and Loren N. Pfeiffer

Citation: Appl. Phys. Lett. 102, 241905 (2013); doi: 10.1063/1.4811708

View online: http://dx.doi.org/10.1063/1.4811708

View Table of Contents: http://apl.aip.org/resource/1/APPLAB/v102/i24

Published by the AIP Publishing LLC.

Additional information on Appl. Phys. Lett.

Journal Homepage: http://apl.aip.org/

Journal Information: http://apl.aip.org/about/about_the_journal

Top downloads: http://apl.aip.org/features/most_downloaded

Information for Authors: http://apl.aip.org/authors

\section{ADVERTISEMENT}
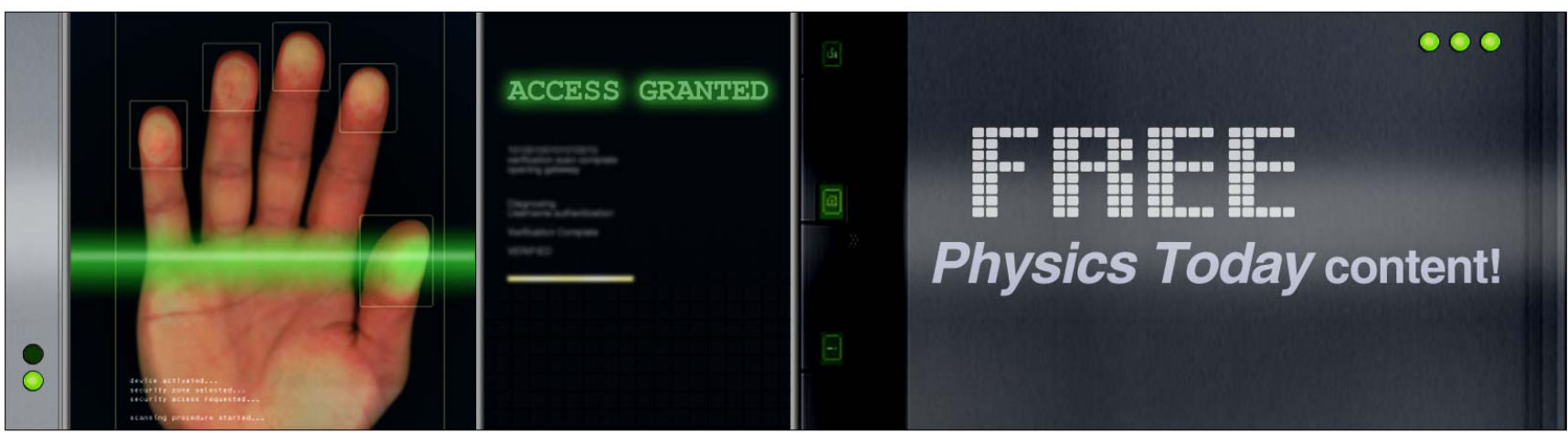


\title{
Counting molecular-beam grown graphene layers
}

\author{
Annette S. Plaut, ${ }^{1}$ Ulrich Wurstbauer, ${ }^{2}$ Aron Pinczuk, ${ }^{2,3}$ Jorge M. Garcia, ${ }^{4}$ \\ and Loren N. Pfeiffer ${ }^{5}$ \\ ${ }^{1}$ School of Physics, University of Exeter, Exeter EX4 4QL, United Kingdom \\ ${ }^{2}$ Department of Physics, Columbia University, New York, New York 10027, USA \\ ${ }^{3}$ Department of Applied Physics and Applied Mathematics, Columbia University, New York, \\ New York 10027, USA \\ ${ }^{4}$ MBE Lab, IMM-Instituto de Microelectronica de Madrid (CNM-CSIC), Madrid, E-28760, Spain \\ ${ }^{5}$ Electrical Engineering Department, Princeton University, New Jersey 08544, USA
}

(Received 29 April 2013; accepted 6 June 2013; published online 19 June 2013)

\begin{abstract}
We have used the ratio of the integrated intensity of graphene's Raman G peak to that of the silicon substrate's first-order optical phonon peak, accurately to determine the number of graphene layers across our molecular-beam (MB) grown graphene films. We find that these results agree well both, with those from our own exfoliated single and few-layer graphene flakes, and with the results of Koh et al. [ACS Nano 5, 269 (2011)]. We hence distinguish regions of single-, bi-, tri-, four-layer, etc., graphene, consecutively, as we scan coarsely across our MB-grown graphene. This is the first, but crucial, step to being able to grow, by such molecular-beam-techniques, a specified number of large-area graphene layers, to order. (C) 2013 AIP Publishing LLC.
\end{abstract}

[http://dx.doi.org/10.1063/1.4811708]

Single-layer graphene is a two-dimensional honeycomb lattice of close-packed carbon atoms, with a linear bandstructure dispersion near its Dirac K points. Its electrons therefore behave as massless Dirac fermions. This leads to unusual transport effects, such as the half-integer quantum Hall effect. ${ }^{1}$ Such unique properties are purely a result of the symmetry of the single-layer graphene lattice. However, the lack of an energy gap between its valence and conduction bands means that single-layer graphene is not the best candidate to form the basis of electronic devices. Hence attention has turned of late to few-layer graphene, where the parabolic band dispersions have tunable bandgaps. ${ }^{2}$

There is thus a great need in the field of graphene research for large-area, few-layer graphene films, comprised of a predetermined number of graphene layers. Molecular beam expitaxy (MBE) has the potential to produce graphene films with such well-controlled characteristics. In order to have control over such growth parameters, one first needs a rapid, sensitive, and non-destructive technique to determine the number of layers grown. In this Letter, we describe how we have employed Raman spectroscopy to determine the number of graphene layers in our few-layer molecular-beam (MB) grown graphene films. This is the first, but crucial, step to being able to grow, by such molecular-beam-techniques, a specified number of large-area graphene layers, on demand.

There are a number of peaks in the Raman spectrum of graphene on $\mathrm{SiO}_{2} / \mathrm{Si}$, deriving both from the graphene itself and its underlying substrate. The silicon substrate produces Raman peaks at energy shifts of 520, 1000, and $1450 \mathrm{~cm}^{-1}$, corresponding to the first-order transverse optical phonon peak (Si-1TO), the $\mathrm{Si}-2 \mathrm{TO}$ and the $\mathrm{Si}-3 \mathrm{TO}$, respectively, whilst the $\mathrm{D}$ peak, at an energy shift of $1350 \mathrm{~cm}^{-1}$, the $\mathrm{G}$ peak at $1600 \mathrm{~cm}^{-1}$ and the $2 \mathrm{D}$, or $\mathrm{G}^{\prime}$, peak at $2700 \mathrm{~cm}^{-1}$ are the main peaks arising from the graphene itself. Although the appearance of the D peak signifies the existence of hexagonal carbon rings, it requires the presence of disorder, such as edges or atomic defects to be activated.
The $\mathrm{G}$ mode is a long-wavelength, doubly degenerate optical mode (the first-order in-plane TO (iTO) and longitudinal optical (LO) phonons) originating from the in-plane bondstretching oscillation of pairs of $\mathrm{sp}^{2}$-hybridised carbon atoms. The 2D mode (also known as $\mathrm{G}^{\prime}$ ) is the second harmonic of the $\mathrm{D}$ mode and requires the participation of two iTO phonons near the Brillouin zone boundary, to be observed.

Up until recently optical contrast ${ }^{3}$ and the shape of the 2D $\left(\mathrm{G}^{\prime}\right)$ Raman line ${ }^{4,5}$ were the only relative quick and convenient means of counting the atomic planes of few-layer graphene. Unfortunately such methods are only applicable up to about 6-layer graphene. ${ }^{3}$ Very recently, new Raman lines have been discovered whose layer-number dependent energy position might be used to probe the number of layers $(n){ }^{6}$ However, the intensities of both these candidates are tens of times smaller than the $\mathrm{G}$ line and they are therefore not particularly accessible. Alternative techniques, such as atomic force microscopy ${ }^{5,7,8}$ are cumbersome, slow, and expensive.

Recently the ratio of the integrated intensity of the Raman G line to that of the silicon 1TO phonon line has been proposed as a reliable, relatively simple alternative, ${ }^{7}$ with particular application for few-layer graphene where $n$ exceeds 4 . This ratio increases monotonically, and in particular, discretely, due to the intensity of the G peak increasing, whilst that of the Si-1TO line decreases, with increasing $n$.

Much of the research on graphene to date has been undertaken on mechanically exfoliated graphene flakes. ${ }^{9}$ Whilst this method produces ultra-high quality graphene crystals, it has not provided the large-area graphene films required for many experiments and applications. Thus, there has been an intense effort to find alternative techniques that produce large-area graphene films, such as thermal decomposition of $\mathrm{SiC},{ }^{10}$ and chemical vapour deposition (CVD) of carbon onto metallic substrates. ${ }^{11,12}$ However, both of these techniques require specific substrates and the graphene 
thereby produced has, typically so far, been of comparatively low mobility. ${ }^{12} \mathrm{MBE}$, on the other hand, has an amazing record-breaking history of growth of III-V semiconductor nanostructures. ${ }^{13}$ This success has mainly been due to its ultra-high vacuum growth environment. MB-growth of graphene has the potential to provide graphene nanostructures with controlled doping on arbitrary substrates. It is also, unlike CVD graphene growth on copper, not restricted to the growth of single graphene layers, and thus has the potential to produce a specified number of large-area graphene layers, on demand. There has, of late, therefore been a push to explore the MB-growth of graphene by a number of groups. ${ }^{14-16}$

We have grown graphene by MB deposition/epitaxy on a selection of substrates ${ }^{15,16}$ and have characterised the resulting graphene films using near edge X-ray absorption fine structure spectroscopy (NEXAFS). This has enabled us to distinguish growth regions on our graphene grown on $\mathrm{Si} /$ $\mathrm{SiO}_{2}$ substrates where, the $\mathrm{sp}^{2}$ carbon bonds are isotropically arranged and other regions where these $\mathrm{sp}^{2}$ bonds lie in a two-dimensional (2D) plane parallel to the substrate. ${ }^{15}$ The former occurs at higher growth rates, whilst the latter forms at lower growth rates. Up until now, the only means to determine the number of graphene layers in the 2D-aligned graphene regions has been by measuring the position-dependent thickness of a thick graphene film $(>30 \mathrm{~nm})$ on a $\mathrm{Si} / \mathrm{SiO}_{2}$ substrate by atomic-force microscopy. ${ }^{15,16}$ In this Letter, we show that by employing the ratio of the integrated intensity of the Raman $G$ line to that of the silicon 1TO phonon line from our 2D-aligned MB-grown graphene films, we have been able to identify, non-destructively, the number of graphene layers present in the region of the sample close to the onset of graphene growth on a $\mathrm{Si} / \mathrm{SiO}_{2}$ substrate. Due to the amorphous nature of the $\mathrm{SiO}_{2}, \mathrm{MB}$ graphene growth does not occur epitaxially, unlike in the case of MB-growth of graphene on hexagonal boron nitride, ${ }^{16}$ where growth is by van der Waals epitaxy. And the thickness of MB-grown graphene films on $\mathrm{SiO}_{2}$ varies across the sample due to the growth geometry within our MB-chamber (Figure 1). The Raman measurements described here enable us to show that the graphene film thickness increases from single-layer graphene, up through bi-layer, tri-layer, and beyond, step-wise

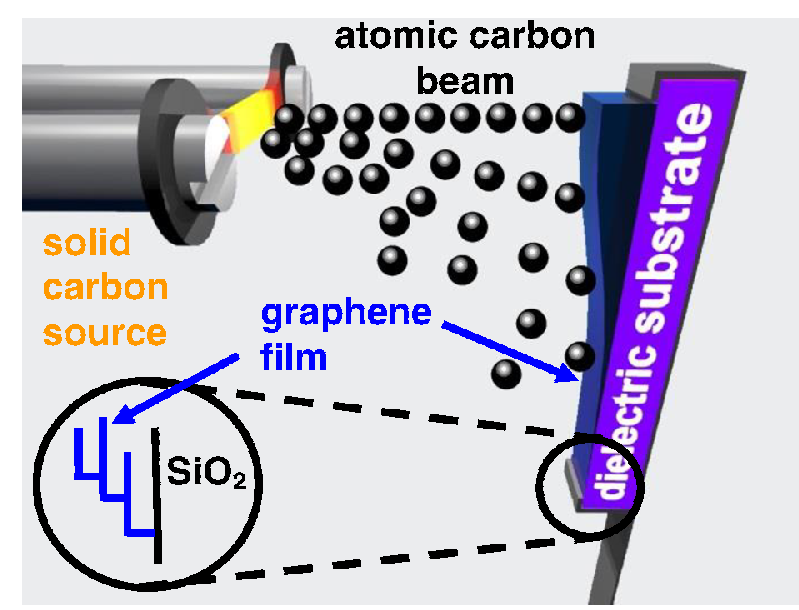

FIG. 1. Schematic of the molecular-beam deposition. Inset: Expanded schematic of the growth of graphene layers on the silicon dioxide substrate. from the onset of graphene growth at the low-growth-rate end of the sample.

The graphene layers were grown on a 300 nm-thick layer of $\mathrm{SiO}_{2}$ on $\mathrm{Si}$ in an ultra-high-vacuum, molecularbeam epitaxy chamber of custom design and construction, equipped with a solid carbon source in close proximity to the substrate, as shown in Figure 1. ${ }^{15,16}$ Due to this close proximity of the substrate to the hot $\left(\sim 2100^{\circ} \mathrm{C}\right)$ carbon source, the substrate heats to $\sim 500^{\circ} \mathrm{C}$ during growth. This growth geometry results in the graphene film thickness depending, in a well-understood manner, on its location on the $25 \mathrm{~mm}$ long $\mathrm{Si} / \mathrm{SiO}_{2}$ substrate. ${ }^{15,16}$

The micro-Raman measurements were taken in a Renishaw inVia Raman microscope, equipped with an adjustable $x-y-z$ stage and a $532 \mathrm{~nm}$ laser, which was focussed to a spot size of $\sim 0.5 \mu \mathrm{m}$. Spectra were excited with a laser power of $2.7 \mathrm{~mW}$.

Figure 2 shows typical Raman spectra from our MBdeposited graphene samples: Figures 2(a) and 2(b) are from the graphene layers, whilst Figure 2(c) shows the first-order optical phonon peak from the silicon substrate. At high growth rates, i.e., from the end of the sample that was closest to the carbon source during growth, we observe the $\mathrm{D}$ $\left(\sim 1350 \mathrm{~cm}^{-1}\right), \mathrm{G}\left(\sim 1600 \mathrm{~cm}^{-1}\right), 2 \mathrm{D}\left(\mathrm{G}^{\prime}\right)\left(\sim 2700 \mathrm{~cm}^{-1}\right)$, the $\mathrm{G}+\mathrm{D}\left(\sim 3000 \mathrm{~cm}^{-1}\right)$ and the $\mathrm{G}+\mathrm{D}^{\prime}\left(\sim 3200 \mathrm{~cm}^{-1}\right)$ Raman peaks. ${ }^{15}$ At these high growth rates, the $\mathrm{D}$ peak can be more intense than the G peak. ${ }^{15}$ Figure 2(a) shows a Raman spectrum typical of MB-grown graphene, deposited at a fairly high growth rate $(4.25 \mathrm{~mm}$ above the onset of graphene growth, which is $16.00 \mathrm{~mm}$ from the sample end that was closest to the carbon source during growth), where the D peak is comparable in size to the $G$ peak and where there is significant Raman intensity between these two lines, which originates from the presence of disordered carbon bonds. ${ }^{17}$ This location on the sample is just within the region where the graphene layers are isotropically oriented. ${ }^{15}$ Figure 2(b) originates from $0.50 \mathrm{~mm}$ above the onset of graphene growth (which is $19.75 \mathrm{~mm}$ from the sample end that was closest to the carbon source during growth), i.e., where the graphene has been deposited at a low growth rate and hence the graphene layers all lie parallel to the $\mathrm{Si} / \mathrm{SiO}_{2}$ substrate surface. ${ }^{15}$ Here, as well as the overall Raman intensity being much reduced due to the fewer number of graphene layers deposited, the intensity of the $\mathrm{D}$ line is also reduced relative to the $\mathrm{G}$ line. In addition, the $\mathrm{D}$ and $\mathrm{G}$ lines are better resolved, due to the absence of disordered carbon bonds, allowing the appearance of the silicon $3 \mathrm{TO}$ phonon line at $1450 \mathrm{~cm}^{-1}$. The intensity scales of all the Raman spectra in Figure 2 are directly comparable, as these spectra were taken under similar experimental conditions. In the Raman spectra of Figures 2(a) and 2(b), however, a linear background between $1249 \mathrm{~cm}^{-1}$ and $1754 \mathrm{~cm}^{-1}$ has been removed.

Following the procedure described in Ref. 7, we have taken Raman spectra along our MB-grown graphene film, in the region where the graphene crystals are aligned in twodimensional planes parallel to the substrate, at $0.125 \mathrm{~mm}$ intervals and hence have determined the ratio of the integrated intensity of the Raman $\mathrm{G}$ line to that of the silicon 1 TO phonon line, $\mathrm{I}(\mathrm{G}) / \mathrm{I}(\mathrm{Si}-1 \mathrm{TO})$, for each spectrum. These ratios are plotted in Figure 3 (black filled circles). For 

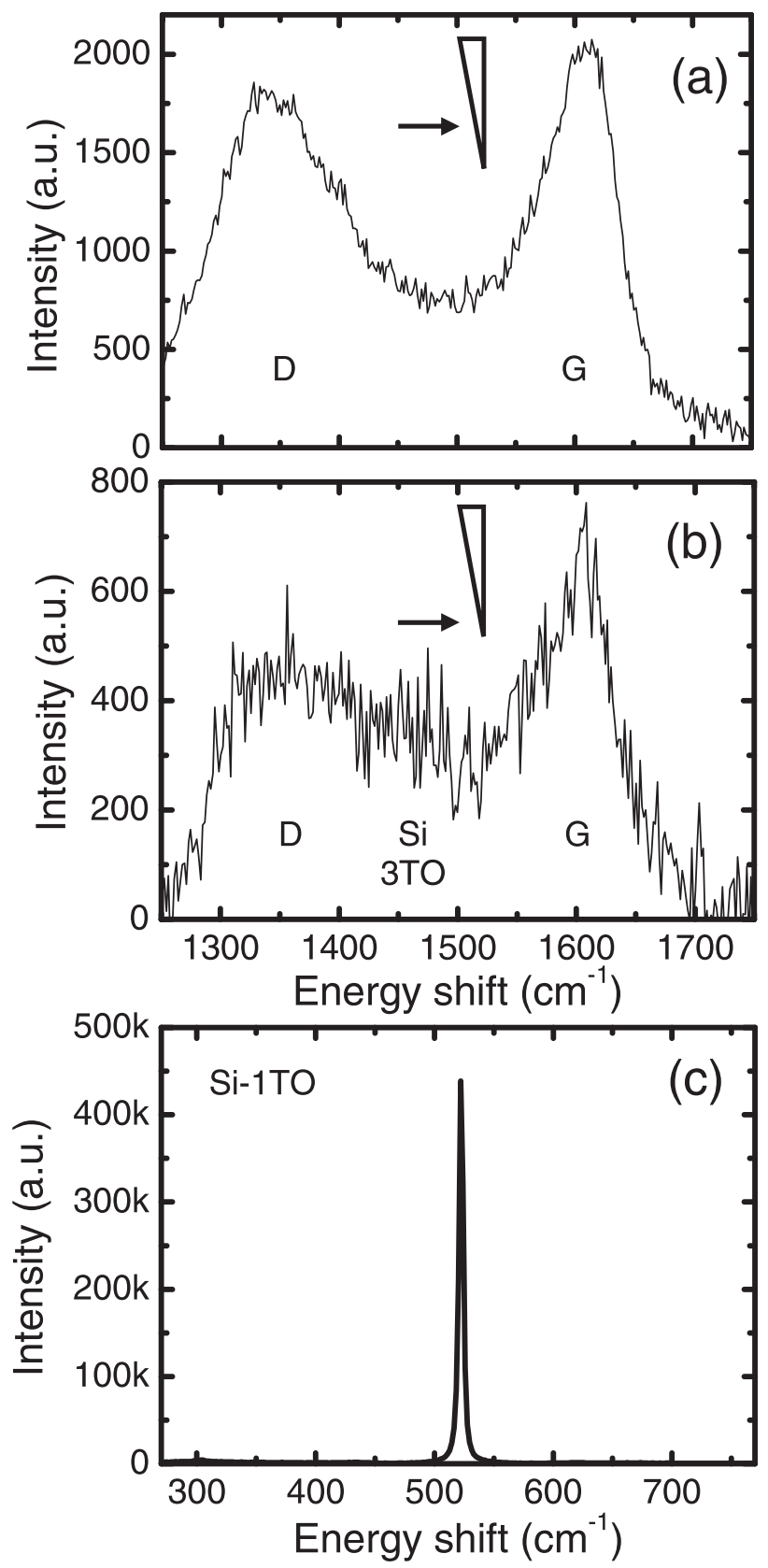

FIG. 2. Typical Raman spectra from (a) isotropically arranged graphene layers, (b) the two-dimensional graphene growth parallel to the $\mathrm{Si} / \mathrm{SiO}_{2}$ substrate surface and (c) the silicon substrate. Note, the different intensity scales in each case, for spectra taken under similar experimental conditions. Insets: Schematics representing the location of the laser beam on the graphene film.

comparison, we also measured, and derived $\mathrm{I}(\mathrm{G}) / \mathrm{I}(\mathrm{Si}-1 \mathrm{TO})$ from the Raman spectra of graphene flakes, fabricated by micromechanical cleavage of highly oriented pyrolytic graphite (HOPG), which we had also deposited on a substrate of $300 \mathrm{~nm}$-thick $\mathrm{SiO}_{2}$ on $\mathrm{Si}$. These results are also plotted in Figure 3 (red open circles), where we have numbered the flakes, in a similar manner to Ref. 7 , in ascending $\mathrm{I}(\mathrm{G}) /$ $\mathrm{I}(\mathrm{Si}-1 \mathrm{TO})$ order.

It is important to note that Ref. 7 clearly states that they find for their exfoliated graphene flakes on $104 \mathrm{~nm}$-thick $\mathrm{SiO}_{2}$ that the $\mathrm{I}(\mathrm{G}) / \mathrm{I}(\mathrm{Si}-1 \mathrm{TO})$ ratios are discrete for $n=1-10$ and can readily be used to determine the number of layers of graphene. However, for their exfoliated graphene flakes on 280 nm-thick $\mathrm{SiO}_{2}$, the ratios of $\mathrm{I}(\mathrm{G}) / \mathrm{I}(\mathrm{Si}-1 \mathrm{TO})$ are less

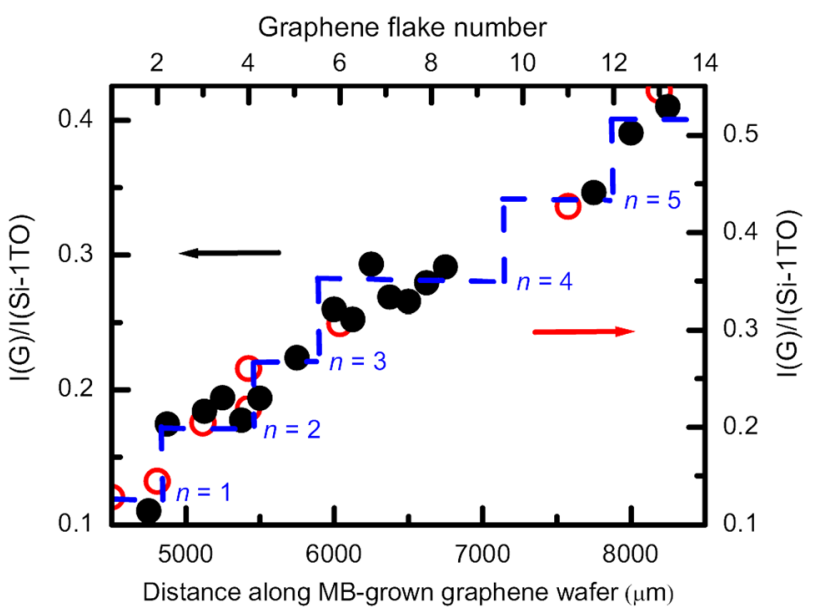

FIG. 3. Ratios of the integrated intensity of the Raman $G$ peak, $I(G)$, and the first-order optical phonon peak of silicon, I(Si-1TO), for molecular-beam grown graphene (black filled circles) and for exfoliated graphene flakes (red open circles). The graphene flakes are numbered in ascending $\mathrm{I}(\mathrm{G}) / \mathrm{I}(\mathrm{Si}-1 \mathrm{TO})$ order. The dashed line is a guide for the eye with the $\mathrm{I}(\mathrm{G}) /$ $\mathrm{I}(\mathrm{Si}-1 \mathrm{TO})$ ratios for the given assigned number of layers $n$ based on the data of Figure 2 of Ref. 7.

discrete, not, they claim, due to $\mathrm{I}(\mathrm{G}) / \mathrm{I}(\mathrm{Si}-1 \mathrm{TO})$ having a weak dependence on $n$, but rather because of the weak colour contrast of graphene on $280 \mathrm{~nm}$-thick $\mathrm{SiO}_{2}$ which makes the identification of regions of homogeneous thickness difficult to identify. Both our exfoliated flakes and our MB-grown graphene are deposited on a 300 nm-thick layer of $\mathrm{SiO}_{2}$ on $\mathrm{Si}$, which clearly is not the most optimal $\mathrm{SiO}_{2}$ thickness to produce the most discrete of jumps in the resulting plot of $\mathrm{I}(\mathrm{G}) / \mathrm{I}(\mathrm{Si}-1 \mathrm{TO})$.

Nevertheless, our data for our exfoliated graphene flakes-red open circles in Figure 3-exhibit a step-like increase in $\mathrm{I}(\mathrm{G}) / \mathrm{I}(\mathrm{Si}-1 \mathrm{TO})$ with exfoliated flake number. We have assigned the number of graphene layers to the individual steps, by the direct comparison of our exfoliated graphene flake data with Figure 2 of Ref. 7. Graphene flake number 1 was also identified as single-layer graphene by the single-Lorentzian lineshape of its $2 \mathrm{D}\left(\mathrm{G}^{\prime}\right)$ Raman line and the significantly greater Raman intensity of this $2 \mathrm{D}$ $\left(\mathrm{G}^{\prime}\right)$ line compared to that of its $\mathrm{G}$ peak. We have also analysed the optical contrast ${ }^{3}$ of our exfoliated graphene flakes and find that the step-like trend of their optical contrast with flake number is almost identical to that shown in Figure 3 for the dependence of $\mathrm{I}(\mathrm{G}) / \mathrm{I}(\mathrm{Si}-1 \mathrm{TO})$ on graphene flake number.

For our MB-grown graphene film-black filled circles in Figure 3-the graphene film thickness increases, with distance along the film, from single-layer graphene, up through bi-layer, tri-layer, and beyond, step-wise from the onset of graphene growth at the low-growth-rate end of the sample, in exactly the same way as that shown for our exfoliated graphene flakes (red open circles in Figure 3).

The discrete nature of the steps in Figure 3 confirms that our MB-grown graphene films are multi-crystalline, ${ }^{15}$ as a continuous film would not exhibit such steps, which clearly correspond to sudden, sharp integer increases in layer number and hence film thickness. These measurements prove that we can grow single and few-layer graphene films by MB-techniques and that by using Raman spectroscopy that 
we can quickly, non-invasively and accurately determine how many layers are present.

Although we have concentrated on results using the Si1TO line, similar results were obtained using the integrated intensity of the $\mathrm{Si}-2 \mathrm{TO}$ line in the intensity ratio calculations. Thus such an approach may possibly be extended to graphene grown on some other substrates-all that is required is that the substrate has its own characteristic Raman line.

In conclusion, we have successfully used this new Raman-based technique, which involves measuring the ratio of the integrated intensity of the graphene $G$ peak to that of the Si first-order optical phonon peak, accurately to determine the number of graphene layers in our MB-grown graphene films on $\mathrm{SiO}_{2} / \mathrm{Si}$ substrates. We have thereby determined that we have indeed grown single-, bi-, tri-, fourlayer, etc. graphene. This is the first, but crucial, step to being able to grow, by such molecular-beam-techniques, a specified number of large-area graphene layers, to order.

Work supported by ONR (N000140610138 and Graphene Muri), EFRC Center for Re-Defining Photovoltaic Efficiency through Molecule Scale Control (award DE-SC0001085), NSF (CHE-0641523), NYSTAR, CSIC-PIF (200950I154), Spanish CAM (Q\&C Light (S2009ESP-1503), Numancia 2 (S2009/ ENE-1477)), and Spanish MEC (ENE2009-14481-C02-02, TEC2011-29120-C05-04, MAT2011-26534).

${ }^{1}$ K. S. Novoselov, A. K. Geim, S. V. Morosov, D. Jiang, M. I. Katsnelson, I. V. Grigorieva, S. V. Dubonos, and A. A. Firsov, Nature 438, 197 (2005); Y. Zhang, Y.-W. Tan, H. L. Stormer, and P. Kim, Nature 438, 201 (2005).

${ }^{2}$ E. V. Castro, K. S. Novoselov, S. V. Morosov, N. M. R. Peres, J. M. B. Lopes dos Santos, J. Nilsson, F. Guinea, A. K. Geim, and A. H. Castro Neto, Phys. Rev. Lett. 99, 216802 (2007); Y. Zhang, T.-T. Tang, C. Girit, Z. Hao, M. C. Martin, A. Zettl, M. F. Crommie, Y. R. Shen, and F. Wang, Nature 459, 820 (2009).

${ }^{3}$ I. Khrapach, F. Withers, T. H. Bointon, D. K. Polyushkin, W. L. Barnes, S. Russo, and M. F. Craciun, Adv. Mater. 24, 2844 (2012).

${ }^{4}$ A. C. Ferrari, J. C. Meyer, V. Scardaci, C. Casiraghi, M. Lazzeri, F. Mauri, S. Piscanec, D. Jiang, K. S. Novoselov, S. Roth, and A. K. Geim, Phys. Rev. Lett. 97, 187401 (2006); Y. Hao, Y. Wang, L. Wang, Z. Ni, Z. Wang, R. Wang, C. K. Koo, Z. Shen, and J. T. L. Thong, Small 6, 195 (2010).
${ }^{5}$ D. Graf, F. Molitor, K. Ensslin, C. Stampfer, A. Jungen, C. Hierold, and L. Wirtz, Nano Lett. 7, 238 (2007).

${ }^{6}$ P. H. Tan, W. P. Han, W. J. Zhao, Z. H. Wu, K. Chang, H. Wang, Y. F. Wang, N. Bonini, N. Marzari, N. Pugno, G. Savini, A. Lombardo, and A. C. Ferrari, Nature Mater. 11, 294 (2012); F. Herziger, P. May, and J. Maultzsch, Phys. Rev. B 85, 235447 (2012).

${ }^{7}$ Y. K. Koh, M.-H. Bae, D. G. Cahill, and E. Pop, ASC Nano 5, 269 (2011). ${ }^{8}$ A. Gupta, G. Chen, P. Joshi, S. Tadigadapa, and P. C. Eklund, Nano Lett. 6, 2667 (2006).

${ }^{9}$ K. S. Novoselev, A. K. Geim, S. V. Morosov, D. Jiang, Y. Zhang, S. V. Dubonos, I. V. Grigorieva, and A. A. Firsov, Science 306, 666 (2004).

${ }^{10}$ C. Berger, Z. Song, T. Li, X. Li, A. Y. Ogbazghi, R. Feng, Z. Dai, A. N. Marchenkov, E. H. Conrad, P. N. First, and W. A. de Heer, J. Phys. Chem. B 108, 19912 (2004).

${ }^{11}$ K. S. Kim, Y. Zhao, H. Jang, S. Y. Lee, J. M. Kim, K. S. Kim, J.-H. Ahn, P. Kim, J.-Y. Choi, and B. H. Hong, Nature 457, 706 (2009); X. Li, W. Cai, J. An, S. Kim, J. Nah, D. Yang, R. Piner, A. Velamakanni, I. Jung, E. Tutuc, S. J. Banerjee, L. Columbo, and R. S. Ruoff, Science 324, 1312 (2009); A. Riena, X. Jia, J. Ho, D. Nezich, H. Son, V. Bulovic, M. S. Dresselhaus, and J. Kong, Nano Lett. 9, 30 (2009).

${ }^{12}$ N. Petrone, C. R. Dean, I. Meric, A. M. van der Zande, P. Y. Huang, L. Wang, D. Muller, K. L. Shephard, and J. Hone, Nano Lett. 12, 2751 (2012).

${ }^{13}$ L. Pfeiffer and K. W. West, Physica E 20, 57 (2003).

${ }^{14}$ A. Al-Temimy, C. Riedl, and U. Starke, Appl. Phys. Lett. 95, 231907 (2009); J. Hackley, D. Ali, J. DiPasquale, J. D. Demaree, and C. J. K. Richardson, Appl. Phys. Lett. 95, 133114 (2009); E. Moreau, S. Godley, F. J. Ferrer, D. Vignaud, X. Wallart, J. Avila, A. C. Acensio, F. Bournel, and J.-J. Gallet, Appl. Phys. Lett. 97, 241907 (2010); J. Park, W. C. Mitchel, L. Grazulis, H. E. Smith, K. G. Eyink, J. J. Boeckl, D. H. Tomich, S. D. Pacley, and J. E. Hoelscher, Adv. Mater. 22, 4140 (2010); F. Maeda and H. Hibino, Jpn. J. Appl. Phys. 49, 04DH13 (2010); J. M. Garcia, R. He, M. P. Jiang, J. Yan, A. Pinczuk, Y. M. Zuev, K. S. Kim, P. Kim, K. Baldwin, K. W. West, and L. N. Pfeiffer, Solid State Commun. 150, 809 (2010); J. Tang, C. Y. Kang, L. M. Li, W. S. Yan, S. Q. Wei, and P. S. Xu, Physica E 43, 1415 (2011); S. K. Jerng, D. S. Yu, Y. S. Kim, J. Ryou, S. Hong, C. Kim, S. Yoon, D. K. Efetov, P. Kim, and S. H. Chun, Phys. Chem. C 115, 4491 (2011); G. Lippert, J. Dabrowski, M. Lemme, C. Marcus, O. Seifarth, and G. Lupina, Phys. Status Solidi B 248, 2619 (2011); Z. Lui, J. Tang, C. Kang, C. Zou, W. Yan, and P. Xu, Solid State Commun. 152, 960 (2012); G. Lippert, J. Dabrowski, Y. Yamamoto, F. Herziger, J. Maultzsch, M. C. Lemme, W. Mehr, and G. Lupina, Carbon 52, 40 (2013).

${ }^{15}$ U. Wurstbauer, T. Schiros, C. Jaye, A. S. Plaut, R. He, A. Rigosi, C. Gutiérrez, D. Fischer, L. N. Pfeiffer, A. N. Pasupathy, A. Pinczuk, and J. M. Garcia, Carbon 50, 4822 (2012).

${ }^{16}$ J. M. Garcia, U. Wurstbauer, A. Levy, L. N. Pfeiffer, A. Pincuk, A. S. Plaut, L. Wang, C. R. Dean, R. Buizza, A. M. Van Der Zande, J. Hone, K. Watanabe, and T. Taniguchi, Solid State Commun. 152, 975 (2012).

${ }^{17}$ A. C. Ferrari and J. Robertson, Phys. Rev. B 61, 14095 (2000); A. C. Ferrari, Solid State Commun. 143, 47 (2007). 